\title{
Algebraic multigrid for stationary and time-dependent partial differential equations with stochastic coefficients
}

\author{
Eveline Rosseel \\ Tim Boonen \\ Stefan Vandewalle \\ Report TW 491, May 2007
}

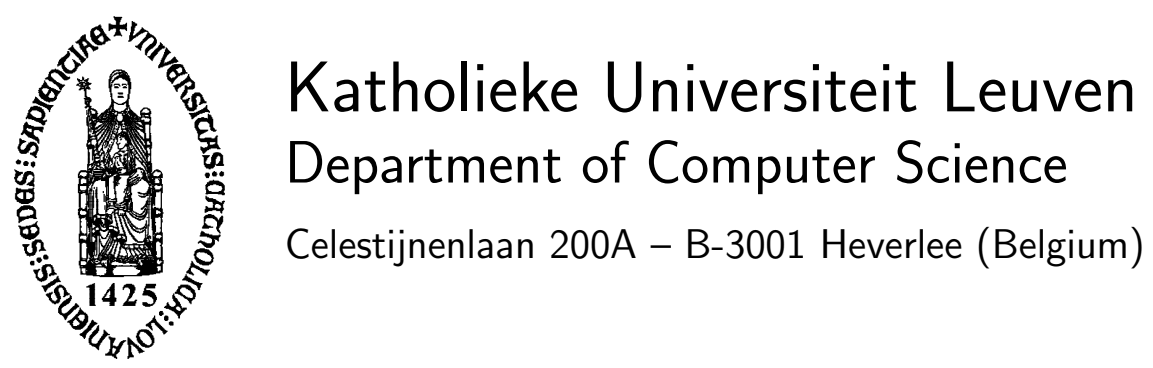




\title{
Algebraic multigrid for stationary and time-dependent partial differential equations with stochastic coefficients
}

\author{
Eveline Rosseel \\ Tim Boonen \\ Stefan Vandewalle \\ Report TW 491, May 2007 \\ Department of Computer Science, K.U.Leuven
}

\begin{abstract}
We consider the numerical solution of time-dependent partial differential equations with random coefficients. A spectral approach, called stochastic finite element method, is used to compute the statistical characteristics of the solution. This method transforms a stochastic partial differential equation into a coupled system of deterministic equations by means of a Galerkin projection onto a generalized polynomial chaos. An algebraic multigrid method is presented to solve the algebraic systems that result after discretization of this coupled system. High-order time integration schemes of an implicit Runge-Kutta type, and spatial discretization on unstructured finite element meshes are considered. The convergence properties of the algebraic multigrid method are demonstrated by a convergence analysis and by numerical tests.
\end{abstract}

Keywords : Algebraic multigrid, Implicit Runge-Kutta time discretization, Partial differential equations with stochastic coefficients.

AMS(MOS) Classification : Primary : 65M55, Secondary : 60H15, 65L06. 


\title{
Algebraic multigrid for stationary and time-dependent partial differential equations with stochastic coefficients*
}

\author{
Eveline Rosseel, Tim Boonen, Stefan Vandewalle \\ Department of Computer Science, Katholieke Universiteit Leuven, \\ Celestijnenlaan 200A, B-3001 Leuven, Belgium
}

\begin{abstract}
We consider the numerical solution of time-dependent partial differential equations with random coefficients. A spectral approach, called stochastic finite element method, is used to compute the statistical characteristics of the solution. This method transforms a stochastic partial differential equation into a coupled system of deterministic equations by means of a Galerkin projection onto a generalized polynomial chaos. An algebraic multigrid method is presented to solve the algebraic systems that result after discretization of this coupled system. High-order time integration schemes of an implicit Runge-Kutta type, and spatial discretization on unstructured finite element meshes are considered. The convergence properties of the algebraic multigrid method are demonstrated by a convergence analysis and by numerical tests.
\end{abstract}

Keywords: PDE with random coefficients, Karhunen-Loève expansion, polynomial chaos, algebraic multigrid, implicit Runge-Kutta time discretization

AMS classification: $65 \mathrm{M} 55,60 \mathrm{H} 15,65 \mathrm{~L} 06$

\section{Introduction}

Randomness in a physical problem can be modelled mathematically by using stochastic partial differential equations (PDE). These may contain some stochastic or random parameters, for example in the coefficients of the differential operator, in the boundary and initial conditions, or in the forcing term. Their solution allows to extract statistical information concerning the solution to the physical model, as required e.g. in uncertainty propagation problems.

The solution of a stochastic PDE can be obtained by statistical or deterministic approaches $[15,32]$. The former are typically based on Monte Carlo simulation techniques, see for example $[20,3]$. Monte Carlo methods are often easy to implement, but rapidly become prohibitively expensive with increasing accuracy demands. Examples of deterministic approaches include perturbation methods [1], Neumann expansion methods [22] and the spectral stochastic finite element method [13, 12]. Perturbation and Neumann expansion methods are restricted to small parameter variances and calculate only a few statistical moments of the solution. These restrictions do not hold for the stochastic finite element

\footnotetext{
${ }^{*}$ This paper presents research results of the Belgian Network DYSCO (Dynamical Systems, Control, and Optimization), funded by the Interuniversity Attraction Poles Programme, initiated by the Belgian State, Science Policy Office. The scientific responsibility rests with its authors.
} 
method, which, in principle, enables to compute the full statistical characteristics of the solution. As such, it provide a valuable alternative to Monte Carlo simulations, see [3] for a comparison.

The stochastic finite element method transforms a stochastic PDE into a system of coupled deterministic PDEs after a projection of the random solution onto a suitable finite dimensional random space. We will use the stochastic finite element approach to discretize the random part of the PDE. For time-dependent PDEs, we will employ an implicit Runge-Kutta (IRK) time integration scheme [14]. For IRK methods the dimension of the linear systems to be solved at each time step is proportional to the number of IRK stages. Multigrid methods are available for IRK discretizations of deterministic parabolic PDEs [29, 4]. In this paper, we extend these methods towards PDEs with random coefficients. In particular, we shall study an algebraic multigrid (AMG) approach, suited for unstructured finite element meshes.

The paper is organized as follows. Section 2 describes the discretization of time-dependent stochastic PDEs by means of the stochastic finite element and implicit Runge-Kutta method. The algebraic multigrid method is presented in Section 3. Its convergence properties are analyzed in Section 4 and further discussed in Section 5. Section 6 addresses some implementation issues. In Section 7, numerical experiments are given to illustrate the convergence behavior. Conclusions are presented in Section 8.

\section{The stochastic model problem and its discretization}

\subsection{A two-dimensional diffusion equation}

We consider a diffusion problem with a random, spatially varying and time-dependent diffusion coefficient $\alpha$, defined over a two-dimensional domain $\mathbf{D}$,

$$
\frac{\partial u(\boldsymbol{x}, t, \omega)}{\partial t}-\nabla \cdot(\alpha(\boldsymbol{x}, t, \omega) \nabla u(\boldsymbol{x}, t, \omega))=b(\boldsymbol{x}, t, \omega),
$$

where $\boldsymbol{x} \in \mathbf{D}, t \in \mathbf{T}=[0, T]$ and $\omega \in \Omega$, a sample space. The latter is part of a complete probability space $(\Omega, \mathcal{F}, \mathcal{E})$ with $\sigma$-algebra $\mathcal{F}$ and probability measure $\mathcal{E}$. Further on, we shall only consider the case of a deterministic source term $b(\boldsymbol{x}, t)$. The method, however, immediately extends to the more general case of a stochastic source term. The coefficient $\alpha(\boldsymbol{x}, t, \omega)$ is a random wave and defined by the mapping $\alpha: \mathbf{D} \otimes \mathbf{T} \otimes \Omega \rightarrow \mathbb{R}$. Model problem (1) is completed with suitable boundary conditions, and initial conditions in the time-dependent case; only deterministic conditions are considered here.

\subsection{Discretization of the random part of the problem}

In order to transform the stochastic PDE (1) into a system of deterministic time-dependent PDEs, we will follow the three step procedure of [33]. First, we express the random inputs by a finite number of random variables; second, the solution is approximated using a finite-term expansion with random basis polynomials, and third, we perform a Galerkin projection onto the set of polynomial basis functions. The second step of this procedure will be addressed in Section 2.2.1; steps one and three will be explained in Sections 2.2.2 and 2.2.3 respectively. 


\subsubsection{Generalized polynomial chaos.}

Consider a Hilbert space $L^{2}(\Omega, \mathcal{F}, \mathcal{E})$ of square integrable functions of $L$ independent random variables $\xi_{i}(\omega)$ on $(\Omega, \mathcal{F}, \mathcal{E})$. Further on we will simply write $\xi_{i}$ instead of $\xi_{i}(\omega)$. A finite dimensional subspace $S$ of $L^{2}(\Omega, \mathcal{F}, \mathcal{E})$ is defined through a set of $Q$ basis functions $\left\{\Psi_{q}\right\}_{q=1, \ldots, Q}$ in the random variables $\xi_{1}, \ldots \xi_{L}$. Let $\xi$ denote a vector containing the random variables $\xi_{1}, \ldots \xi_{L}$. The space $S$ is equipped with an inner product defined by $\langle a(\xi) b(\xi)\rangle=$ $\int_{\Omega} a(\xi) b(\xi) w(\xi) d \xi$, with $w(\xi)$ denoting the probability density corresponding to $\xi$. This inner product actually corresponds to an expectation its arguments. Several approaches have been proposed to construct $S$, e.g. $[13,2,33,30]$. Here, we shall employ an orthonormal basis of multivariate polynomials $\Psi_{l}$ that are globally defined in each random variable $\xi_{i}$. These multivariate polynomials are built as a product of univariate polynomials $\left\{\varphi_{m_{i}}\right\}_{i=1, \cdots, L}$ of degree $m_{i}$ in $\xi_{i}$ and orthonormal w.r.t. the probability measure corresponding to $\xi_{i}$.

Two criteria are often considered to determine the basis functions. One may limit the total degree of the polynomial to a given value $P$, i.e., $\sum_{i=1}^{L} m_{i} \leq P$. The total number of basis functions, $Q$, is then given by $(L+P) ! / L ! P ![16]$. Alternatively, one may limit the degrees of the univariate factors separately, i.e. $m_{i} \leq p_{i}, i=1, \cdots, L$, for a given set of $p_{i}$-values. In this case $Q=\prod_{i=1}^{L}\left(p_{i}+1\right)[3]$.

Using the first criterion, a so-called generalized polynomial chaos basis $[33,15]$ can be constructed. The univariate polynomials are chosen from the Wiener-Askey scheme according to the probability distributions of the random variables $\xi_{i}$. The second criterion can be used to create an alternative set of basis functions $\left\{\Psi_{q}^{\prime}\right\}[2,3,21]$, which possess a double orthogonality property,

$$
\left\langle\Psi_{j}^{\prime} \Psi_{k}^{\prime}\right\rangle=\delta_{j, k} \quad \text { and } \quad\left\langle\xi_{i} \Psi_{j}^{\prime} \Psi_{k}^{\prime}\right\rangle=\zeta_{i j k} \delta_{j, k}
$$

with $\zeta_{i j k}$ a constant and $\delta_{j, k}$ the Kronecker delta. This property allows to transform a linear stochastic PDE into a system of uncoupled deterministic PDEs.

Having specified an appropriate random basis, the solution $u(\boldsymbol{x}, t, \omega)$ can be approximated by a linear combination of basis functions with deterministic coefficients $u_{q}(\boldsymbol{x}, t)$. When the basis functions are collected in the column vector $\Psi$ and the coefficients in a column vector $\boldsymbol{u}(\boldsymbol{x}, t)$, we can write

$$
u(\boldsymbol{x}, t, \omega) \approx \sum_{q=1}^{Q} u_{q}(\boldsymbol{x}, t) \Psi_{q}(\xi)=\Psi^{T} \boldsymbol{u}(\boldsymbol{x}, t)
$$

\subsubsection{Discretization of random inputs}

The random inputs are typically discretized either by a generalized polynomial chaos expansion approach similar to (3), see e.g. [11, 34], or by a Karhunen-Loève (KL) expansion [17]. The former leads to an approximation of the form

$$
\alpha(\boldsymbol{x}, t, \omega) \approx \sum_{i=1}^{Q} \alpha_{i}(\boldsymbol{x}, t) \Psi_{i}(\xi) \quad \text { with } \quad \alpha_{i}(\boldsymbol{x}, t)=\frac{\left\langle\alpha(\boldsymbol{x}, t, \omega) \Psi_{q}\right\rangle}{\left\langle\Psi_{q}^{2}\right\rangle} .
$$

We will apply this type of discretization to model random inputs with a lognormal marginal distribution. Analytical expressions for the corresponding $\alpha_{i}(\boldsymbol{x}, t)$ coefficients are given in [10]. 
The truncated Karhunen-Loève expansion approximates a random wave $\alpha(\boldsymbol{x}, t, \omega)$ as

$$
\alpha(\boldsymbol{x}, t, \omega) \approx \alpha_{1}(\boldsymbol{x}, t)+\sum_{i=1}^{L} \alpha_{i+1}(\boldsymbol{x}, t) \xi_{i}(\omega) .
$$

The function $\alpha_{1}(\boldsymbol{x}, t)$ corresponds to the mean of $\alpha(\boldsymbol{x}, t, \omega)$. The functions $\alpha_{i+1}(\boldsymbol{x}, t)$ are eigenfunctions of the covariance function $C_{\alpha \alpha}\left(\boldsymbol{x}_{1}, t_{1} ; \boldsymbol{x}_{2}, t_{2}\right)$, scaled by the square root of the corresponding eigenvalues. The random variables $\xi_{i}$ are uncorrelated random variables with zero mean and unit variance [19]. We assume that these random variables are independent. Note that $L+1$ terms are needed to express a random input in a $L$-dimensional random space by a Karhunen-Loève expansion, in comparison to $Q$ terms in case of a chaos expansion. Hence, a chaos expansion will only be used when the KL-expansion is difficult to compute.

\subsubsection{Galerkin approach}

The stochastic PDE (1) can be converted into a system of deterministic PDEs for the unknown coefficients $u_{q}(\boldsymbol{x}, t)$ that appear in (3). This is done by replacing $\alpha(\boldsymbol{x}, t, \omega)$ by its approximation (4) or (5), by inserting the right-handside of (3) into the PDE, and by imposing orthogonality of the resulting residual w.r.t. the chosen polynomial chaos. This results in

$$
C_{1} \frac{\partial \boldsymbol{u}(\boldsymbol{x}, t)}{\partial t}-\sum_{i=1}^{L^{*}} C_{i} \nabla \cdot\left[\alpha_{i}(\boldsymbol{x}, t) \nabla \boldsymbol{u}(\boldsymbol{x}, t)\right]=b(\boldsymbol{x}, t) c,
$$

with the vector $c=\langle\Psi\rangle$ and the matrices $C_{i}$ defined as

$$
\begin{aligned}
C_{i} & =\left\langle\xi_{i-1} \Psi \Psi^{T}\right\rangle & i=1, \ldots, L^{*}:=L+1 & \text { if } \alpha(\boldsymbol{x}, t, \omega) \text { represented by a KL-expansion } \\
& =\left\langle\Psi_{i} \Psi \Psi^{T}\right\rangle & i=1, \ldots, L^{*}:=Q & \text { if } \alpha(\boldsymbol{x}, t, \omega) \text { represented by a chaos expansion }
\end{aligned}
$$

and $\xi_{0}=1$. The matrix $C_{1}$ equals the identity matrix $I_{Q}$ of dimension $Q$ if the polynomial chaos functions are suitably normalized. Analytical expressions for $\left\langle\xi_{i} \Psi \Psi^{T}\right\rangle$ can be found in [25]; in Appendix we present expressions for $\left\langle\Psi_{i} \Psi \Psi^{T}\right\rangle$, see Eq. (40).

Remark 2.1. In case of a double orthogonal polynomial chaos, the PDEs are uncoupled. Indeed each matrix $C_{i}$ is diagonal due to the orthogonality properties (2). However, the resulting number of PDEs becomes rapidly too large for practical purposes when the number of random variables or the degree of the polynomials increases.

Remark 2.2. The uniqueness and existence of the solution to (6) can be proved from the LaxMilgram lemma under certain conditions on the stochastic parameters, as detailed in $[2,3]$.

\subsection{Spatial finite element discretization}

We shall use classical finite elements for spatial discretization, and assume that each of the deterministic coefficients is discretized on the same mesh, with the same (number of) elements. Hence, each $u_{q}(\boldsymbol{x}, t)$ for $q=1, \ldots, Q$, is approximated as a linear combination of the form $u_{q}(\boldsymbol{x}, t) \approx \sum_{n=1}^{N} u_{q, n}(t) s_{n}(\boldsymbol{x})$, in terms of $N$ basis functions $s_{n}(\boldsymbol{x})$. The coefficients are grouped together in the vectors $\boldsymbol{b}(t), \boldsymbol{u}_{q}(t) \in \mathbb{R}^{N}$. The discretization of (6) can be written 
compactly after introduction of a set of $L^{*}$ deterministic stiffness matrices $K_{i} \in \mathbb{R}^{N \times N}$ defined as $K_{i}=\mathcal{K}\left(\alpha_{i}(\boldsymbol{x}, t)\right)$ with

$$
\left[\mathcal{K}\left(a_{i}(\boldsymbol{x}, t)\right)\right]_{k l}=\int_{\mathbf{D}} a_{i}(\boldsymbol{x}, t) \nabla s_{k}(\boldsymbol{x}) \cdot \nabla s_{l}(\boldsymbol{x}) d \boldsymbol{x} \quad \text { and } \quad i=1, \ldots, L^{*} ; k, l=1, \ldots, N .
$$

After spatial discretization, the stochastic finite element method yields a system of ordinary differential equations (ODE),

$$
C_{1} \otimes M \frac{d u(t)}{d t}+\sum_{i=1}^{L^{*}} C_{i} \otimes K_{i} u(t)=c \otimes \boldsymbol{b}(t) \quad \text { with } u(t)=\left[\begin{array}{c}
\boldsymbol{u}_{1}(t) \\
\vdots \\
\boldsymbol{u}_{Q}(t)
\end{array}\right] \text { and } \boldsymbol{u}_{q}(t) \in \mathbb{R}^{N}
$$

Here, $M \in \mathbb{R}^{N \times N}$ is the mass matrix defined as $[M]_{k l}=\int_{D} s_{k}(\boldsymbol{x}) s_{l}(\boldsymbol{x}) d \boldsymbol{x}$, and the matrices $C_{i} \in \mathbb{R}^{Q \times Q}$ and the vector $c \in \mathbb{R}^{Q}$ are defined by (7) or (8). Formulation (10) assumes that the $Q \cdot N$ unknowns are ordered blockwise per $q$ value, i.e., with each block corresponding to the $N$ scalar unknowns associated with one particular $\boldsymbol{u}_{q}(\boldsymbol{x}, t)$ function. Alternatively, the unknowns can be ordered blockwise per grid point. Each block will then correspond to the $Q$ unknowns associated with one particular $s_{n}(\boldsymbol{x})$ function. The second formulation leads to the following ODE system,

$$
M \otimes C_{1} \frac{d \tilde{u}(t)}{d t}+\sum_{i=1}^{L^{*}} K_{i} \otimes C_{i} \tilde{u}(t)=\boldsymbol{b}(t) \otimes c \quad \text { with } \tilde{u}(t)=\left[\begin{array}{c}
\tilde{\boldsymbol{u}}_{1}(t) \\
\vdots \\
\tilde{\boldsymbol{u}}_{N}(t)
\end{array}\right] \text { and } \tilde{\boldsymbol{u}}_{n}(t) \in \mathbb{R}^{Q}
$$

\subsection{Time discretization}

We consider time discretization by an implicit Runge-Kutta method [14]. In order to introduce some notation, we shall recall the basic formula of such a method, as applied to an ODE of the form, $\frac{d u}{d t}=f(t, u)$. An implicit Runge-Kutta method computes an approximation $u_{m+1}$ to the solution $u\left(t_{m+1}\right)$ at time $t_{m+1}$ from an approximation $u_{m}$ at time $t_{m}$. To this end, it introduces a number of auxiliary variables $x_{j}, j=1, \ldots, s$, called stage values or stage vectors, at times $t_{m}+c_{j} \Delta t$ with $\Delta t=t_{m+1}-t_{m}$. The procedure corresponds to the following set of equations,

$$
\begin{aligned}
u_{m+1} & =u_{m}+\Delta t \sum_{j=1}^{s} b_{j} f\left(t_{m}+c_{j} \Delta t, x_{j}\right), \\
x_{i} & =u_{m}+\Delta t \sum_{j=1}^{s} a_{i j} f\left(t_{m}+c_{j} \Delta t, x_{j}\right) \quad i=1, \ldots, s .
\end{aligned}
$$

Eq. (12) expresses $u_{m+1}$ as an update to $u_{m}$ in terms of the stage values $\left\{x_{i}\right\}_{i=1, \ldots, s}$. Eq. (13) describes the system of equations to be solved in order to compute the stages values. The method is fully characterized by the parameters $A_{\text {irk }}=\left[a_{i j}\right], b_{\text {irk }}=\left[b_{1} \ldots b_{s}\right]^{T}$ and $c_{\text {irk }}=\left[c_{1} \ldots c_{s}\right]^{T}$. Equations (12) and (13) are often rewritten in terms of the stage value increments $\Delta x_{j}:=x_{j}-u_{m}$,

$$
\begin{aligned}
u_{m+1} & =u_{m}+\left[\Delta x_{1} \cdots \Delta x_{s}\right] A_{\mathrm{irk}}^{-1} b_{\mathrm{irk}}, \\
\Delta x_{i} & =\Delta t \sum_{j=1}^{s} a_{i j} f\left(t_{m}+c_{j} \Delta t, u_{m}+\Delta x_{j}\right) \quad i=1, \ldots, s .
\end{aligned}
$$


We will apply the IRK method to system (11). The approximation at time $t_{m+1}$ to the solution $\tilde{u}\left(t_{m+1}\right)$ will be represented as $u_{m+1}$. Formulation (14)-(15) is used with stage vector increments denoted simply as $x_{j}, j=1, \ldots, s$. They are grouped together into a long vector $x \in \mathbb{R}^{N Q s}$, where the increments are numbered first along the spatial dimension, then along the random dimension and finally according to the stages.

When the coefficient $\alpha(\boldsymbol{x}, \omega)$ is time-independent, the system (15) discretizing (11) becomes

$$
\left(M \otimes C_{1} \otimes I_{s}+\Delta t \sum_{i=1}^{L^{*}} K_{i} \otimes C_{i} \otimes A_{\mathrm{irk}}\right) x=\widetilde{b},
$$

with $\widetilde{b}$ a known vector depending on $u_{m}$ and on the right-handside of (11),

$$
\widetilde{b}=\Delta t\left(I_{N Q} \otimes A_{\mathrm{irk}} P^{T}\left(\left[\begin{array}{c}
\boldsymbol{b}\left(t_{m}+c_{1} \Delta t\right) \\
\vdots \\
\boldsymbol{b}\left(t_{m}+c_{s} \Delta t\right)
\end{array}\right] \otimes c\right)-\sum_{i=1}^{L^{*}} K_{i} \otimes C_{i} \otimes A_{\mathrm{irk}}\left[u_{m} \otimes \mathbf{1}_{s}\right]\right)
$$

and $\mathbf{1}_{s}=[1 \ldots 1]^{T} \in \mathbb{R}^{s}$. The matrix $P^{T}$ is such that it permutes the rows of the vector it multiplies so that all variables are grouped in the same order as the unknowns $x$.

In case of a time-dependent stochastic coefficient $\alpha(\boldsymbol{x}, t, \omega)$, each of the elements of the stiffness matrices $K_{i}(9)$ is time-dependent. According to Eq. (15), every stiffness matrix $K_{i}(t)$ is evaluated at $s$ time positions $t=t_{m}+c_{j} \Delta t, j=1, \ldots, s$. This leads to a total of $L^{*} \cdot s$ stiffness matrices at each time step. Applying (15) yields the following system to be solved for the stage vector increments,

$$
\begin{array}{r}
\left(\left(M \otimes C_{1} \otimes I_{s}\right)+\Delta t\left[\sum_{i=1}^{L^{*}} K_{i}\left(t_{m}+c_{1} \Delta t\right) \otimes C_{i} \otimes A_{\mathrm{irk}}(:, 1) \ldots\right.\right. \\
\left.\left.\sum_{i=1}^{L^{*}} K_{i}\left(t_{m}+c_{s} \Delta t\right) \otimes C_{i} \otimes A_{\mathrm{irk}}(:, s)\right] P\right) x=B .
\end{array}
$$

Matrix $P$ is a $N Q s \times N Q s$ permutation matrix. It permutes the columns of the matrix that it is multiplied with so that consecutive IRK stages are grouped together in blocks of $s$ columns. In the remainder of the paper, the multigrid formulation and analysis is presented for timeindependent $\alpha(\boldsymbol{x}, \omega)$. The extension to the general case of $\alpha(\boldsymbol{x}, t, \omega)$ is straightforward.

\section{Algebraic multigrid for the stochastic finite element method}

Next, we present an algebraic multigrid (AMG) method to solve the stochastic finite element discretizations (16) or (18). We will also consider the easier case of a stationary, i.e., timeindependent problem. In that case, the discretization reads

$$
\sum_{i=1}^{L^{*}} C_{i} \otimes K_{i} u=b \quad \text { with } \quad u=\left[\begin{array}{c}
\boldsymbol{u}_{1} \\
\vdots \\
\boldsymbol{u}_{Q}
\end{array}\right], \quad \boldsymbol{u}_{q} \in \mathbb{R}^{N} \text { and } \quad b=c \otimes \boldsymbol{b} .
$$

The basis of the method is the classical multigrid iteration as shown in Algorithm 1. The algorithm uses a hierarchy of $K$ levels, $k=1, \ldots, K$, with $A_{K} u_{K}=b_{K}$ the PDE discretization 


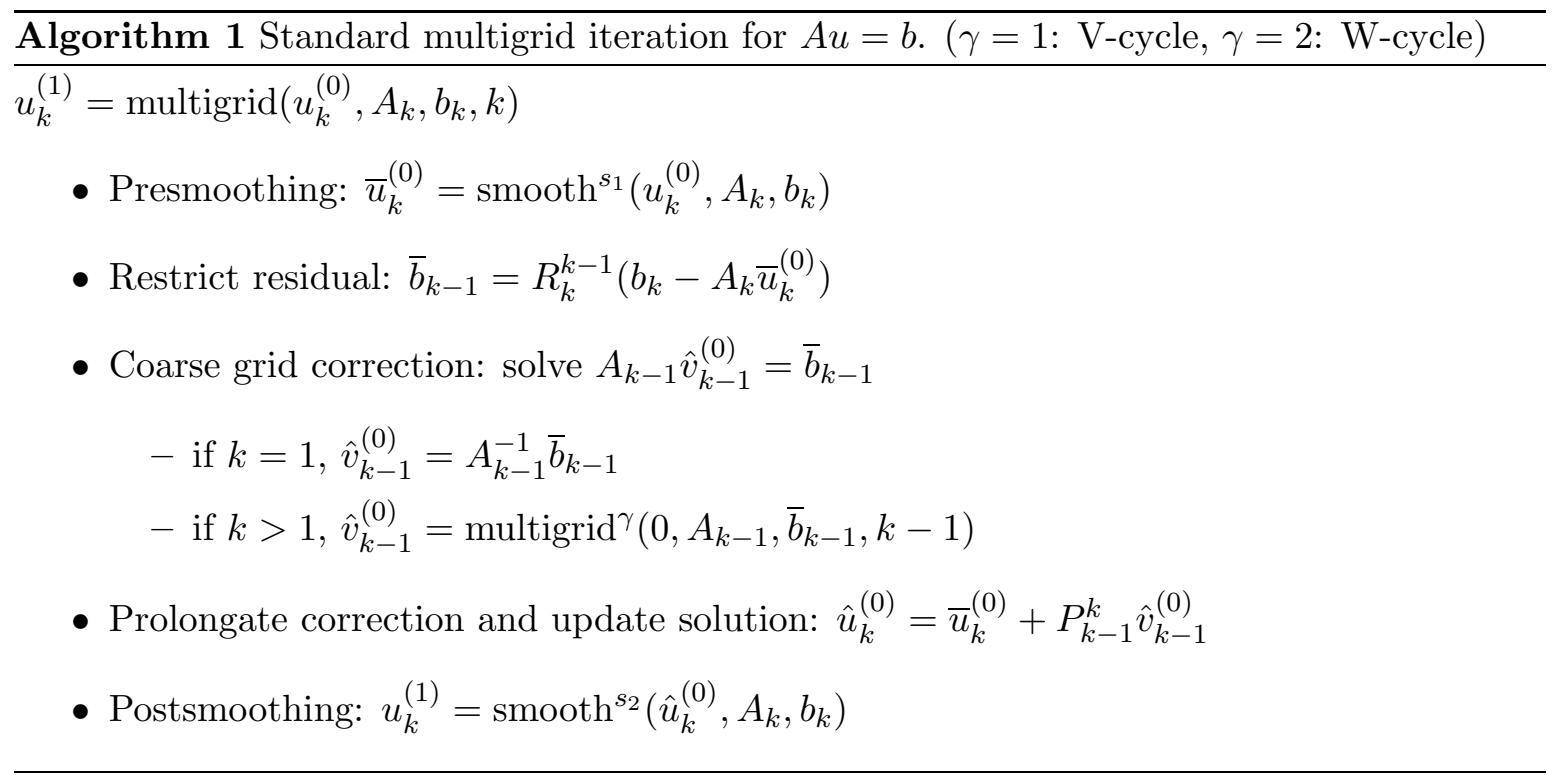

on the given (fine) mesh. The recursion scheme is determined by a parameter $\gamma$; for example the case $\gamma=1$ is called a V-cycle, the case $\gamma=2$ a W-cycle. An AMG method requires a setup phase to algebraically construct the restriction and prolongation operators, $R_{k}^{k-1}$ and $P_{k-1}^{k}, k=2, \ldots, K$. The coarse level operators $A_{k-1}, k=2, \ldots, K$, are assembled by using the Galerkin principle [27], i.e., $A_{k}=R_{k+1}^{k} A_{k+1} P_{k}^{k+1}$. To construct an AMG method for stochastic finite element and implicit Runge-Kutta discretizations, the AMG components are built so that all unknowns per spatial node are updated together. A block smoother will be used, and prolongation and restriction operators will have a tensor structure.

\subsection{A smoother for the stochastic finite element method}

We suggest to use a block lexicographic Gauss-Seidel smoothing method. More precisely, one smoothing step consists of a loop over all spatial nodes, in which all random and IRK unknowns per node are updated simultaneously. Hence, every iteration involves a sequence of $N$ local solves of a linear system. The local system at node $n$ corresponds to the $Q s \times Q s$ system

$$
\begin{aligned}
& \left(M[n, n] C_{1} \otimes I_{s}+\Delta t \sum_{i=1}^{L^{*}} K_{i}[n, n] C_{i} \otimes A_{\text {irk }}\right) x[n]= \\
& \widetilde{b}[n]-\sum_{m \neq n}\left(M[n, m] C_{1} \otimes I_{s}+\Delta t \sum_{i=1}^{L^{*}} K_{i}[n, m] C_{i} \otimes A_{\text {irk }}\right) x[m],
\end{aligned}
$$

with $x[n] \in \mathbb{R}^{Q s}$ the unknowns associated with node $n$. For stationary problems, the local system simplifies to the $Q \times Q$ system

$$
\sum_{i=1}^{L^{*}} K_{i}[n, n] C_{i} u[n]=b[n]-\sum_{m \neq n} \sum_{i=1}^{L^{*}} K_{i}[n, m] C_{i} u[m] .
$$


The block Gauss-Seidel iteration step can be written as a linear iteration based on a matrix splitting of the stiffness matrices $K_{i}, K_{i}=K_{i}^{+}+K_{i}^{-}\left(i=1, \ldots, L^{*}\right)$, and the mass matrix $M$, $M=M^{+}+M^{-}$. Here, $K_{i}^{+}$and $M^{+}$are the lower triangular parts of $K_{i}$ and $M$ respectively. The block Gauss-Seidel iteration in the $\nu$ th iteration step can then be formulated as

$$
\begin{aligned}
&\left(M^{+} \otimes C_{1} \otimes I_{s}\right.\left.+\Delta t \sum_{i=1}^{L^{*}} K_{i}^{+} \otimes C_{i} \otimes A_{\text {irk }}\right) x^{(\nu+1)}= \\
& \widetilde{b}-\left(M^{-} \otimes C_{1} \otimes I_{s}+\Delta t \sum_{i=1}^{L^{*}} K_{i}^{-} \otimes C_{i} \otimes A_{\text {irk }}\right) x^{(\nu)} .
\end{aligned}
$$

\subsection{Multigrid hierarchy and intergrid transfer operators}

We suggest to derive the multigrid hierarchy from the dominant term in (4) and (5), i.e., from the stiffness matrix of the averaged deterministic problem. This is the PDE that results from the stochastic PDE by replacing all the random parameters by their mean value. Such a hierarchy can be derived by using a classical AMG strategy applied to the stiffness matrix $K_{1}$. Suppose $P_{d}$ is a prolongation operator constructed for $K_{1}$, then tensor prolongation and restriction operators for (16), denoted as $\mathcal{P}$ and $\mathcal{R}$ respectively, are built as

$$
\mathcal{P}=P_{d} \otimes I_{Q} \otimes I_{s} \quad \text { and } \quad \mathcal{R}=P_{d}^{T} \otimes I_{Q} \otimes I_{s} .
$$

This simplifies in the stationary case to

$$
\mathcal{P}=I_{Q} \otimes P_{d} \quad \text { and } \quad \mathcal{R}=I_{Q} \otimes P_{d}^{T} .
$$

Note that all random and IRK unknowns associated with a particular spatial node are prolongated and restricted in a decoupled way. Only the spatial dimension is coarsened; the stochastic and time discretization is kept unaltered throughout this multigrid hierarchy. The coarse grid operator, denoted as $\mathcal{A}^{H}$, is deduced from (24) and (23) by using the Galerkin principle. That is, the coarse grid operator corresponds to $\mathcal{R} A^{h} \mathcal{P}$ with $\mathcal{A}^{h}$ the fine grid operator. Thus applying formulas (24)-(23) to Eq. (19)-(16) results in

$$
\mathcal{A}^{H}=P_{d} M P_{d}^{T} \otimes C_{1} \otimes I_{s}+\Delta t \sum_{i=1}^{L^{*}} P_{d} K_{i} P_{d}^{T} \otimes C_{i} \otimes A_{\text {irk }}
$$

for the time-dependent case. For the time-independent case we have

$$
\mathcal{A}^{H}=\sum_{i=1}^{L^{*}} C_{i} \otimes P_{d}^{T} K_{i} P_{d} .
$$

\section{Convergence analysis}

Using Fourier analysis [5, 27], valuable insights in the convergence behavior of geometric multigrid methods can be obtained. A local Fourier analysis of geometric multigrid for stochastic, stationary PDEs can be found in [21]. This analysis cannot directly be applied to algebraic multigrid methods. Instead, the methodology from $[29,4]$ is followed.

Our analysis for stationary and time-dependent problems as will be detailed in Sections 4.1 and 4.2 respectively, is restricted to the case of $L^{*}=2$. This corresponds to a diffusion coefficient modelled as a random variable. In Section 5, the extension to the general case, $L^{*}>2$, is discussed. 


\subsection{Stationary problems}

First, we derive some results for the block smoother. We start from the error iteration,

$$
e^{(\nu+1)}=S e^{(\nu)} \quad \text { with } \quad S=-\left(C_{1} \otimes K_{1}^{+}+C_{2} \otimes K_{2}^{+}\right)^{-1}\left(C_{1} \otimes K_{1}^{-}+C_{2} \otimes K_{2}^{-}\right),
$$

and $e^{(\nu)}=u^{\text {exact }}-u^{(\nu)}$ the error at iteration step $\nu$. The asymptotic convergence is characterized by the spectral radius of the iteration operator $S$, denoted by $\rho(S)$. Assume the random basis functions $\Psi_{1}, \ldots, \Psi_{Q}$ are normalized in such a way that $C_{1}$ equals the identity matrix $I_{Q}$. The matrix $C_{2}$ is a real symmetric matrix (7)-(8) with eigenvalue decomposition $C_{2}=V_{C_{2}} \Lambda_{C_{2}} V_{C_{2}}^{T}$. Applying the similarity transform $V_{C_{2}} \otimes I_{N}$ to $S$ leads to

$$
\begin{aligned}
\sigma(S) & =\sigma\left(V_{C_{2}}^{T} \otimes I_{N} \cdot S \cdot V_{C_{2}} \otimes I_{N}\right) \\
& =\sigma\left(-\left(I_{Q} \otimes K_{1}^{+}+\Lambda_{C_{2}} \otimes K_{2}^{+}\right)^{-1}\left(I_{Q} \otimes K_{1}^{-}+\Lambda_{C_{2}} \otimes K_{2}^{-}\right)\right) \\
& =\bigcup_{q=1}^{Q} \sigma\left(-\left(K_{1}^{+}+\lambda_{q} K_{2}^{+}\right)^{-1}\left(K_{1}^{-}+\lambda_{q} K_{2}^{-}\right)\right) \quad \text { with } \quad \lambda_{q} \in \sigma\left(C_{2}\right) \\
& =\bigcup_{q=1}^{Q} \sigma\left(\hat{S}\left(\lambda_{q}\right)\right),
\end{aligned}
$$

with $\hat{S}$ a matrix-valued function defined as

$$
\hat{S}(r)=-\left(K_{1}^{+}+r K_{2}^{+}\right)^{-1}\left(K_{1}^{-}+r K_{2}^{-}\right) .
$$

Thus the asymptotic convergence factor of block Gauss-Seidel is given by

$$
\rho(S)=\max _{\lambda_{q} \in \sigma\left(C_{2}\right)} \rho\left(\hat{S}\left(\lambda_{q}\right)\right) .
$$

To characterize the convergence properties of a two-level multigrid cycle, we define the matrixvalued function $\hat{T}(r)$,

$$
\hat{T}(r)=(\hat{S}(r))^{s_{2}}\left(I_{N}-P_{d}\left(P_{d}^{T}\left(K_{1}+r K_{2}\right) P_{d}\right)^{-1} P_{d}^{T}\left(K_{1}+r K_{2}\right)\right)(\hat{S}(r))^{s_{1}},
$$

with $\hat{S}(r)$ defined by $(26)$, and with $s_{1}, s_{2}$ the number of pre- and postsmoothing iterations. An analogous derivation as above shows that the asymptotic convergence factor of the twolevel cycle can be determined from the spectral radius of the corresponding iteration matrix $T$ as

$$
\rho(T)=\max _{\lambda_{q} \in \sigma\left(C_{2}\right)} \rho\left(\hat{T}\left(\lambda_{q}\right)\right) .
$$

Formulas (27) and (28) allow the following intuitive interpretation. The convergence for the stationary stochastic finite element discretization with $L^{*}=2$, equals the worst convergence of the corresponding Gauss-Seidel or multigrid method, applied to a set of deterministic problems of the form

$$
\left(K_{1}+\lambda_{q} K_{2}\right) u=b \quad \text { with } \quad \lambda_{q} \in \sigma\left(C_{2}\right)
$$




\subsection{Time-dependent problems}

The error iteration of the block Gauss-Seidel smoother (22) for $C_{1}=I_{Q}$ and $L^{*}=2$, is given by

$$
\begin{aligned}
& \left(M^{+} \otimes I_{Q s}+\Delta t\left(K_{1}^{+} \otimes I_{Q}+K_{2}^{+} \otimes C_{2}\right) \otimes A_{\text {irk }}\right) e^{(\nu+1)}= \\
& \quad-\left(M^{-} \otimes I_{Q s}+\Delta t\left(K_{1}^{-} \otimes I_{Q}+K_{2}^{-} \otimes C_{2}\right) \otimes A_{\text {irk }}\right) e^{(\nu)}
\end{aligned}
$$

with corresponding iteration matrix denoted as $S$. This matrix can be decoupled by applying the similarity transform $I_{N} \otimes V_{C_{2}} \otimes V_{\text {irk }}$, with $V_{\text {irk }}$ resulting from the eigenvalue decomposition $A_{\text {irk }}=V_{\text {irk }} \Lambda_{\text {irk }} V_{\text {irk }}^{-1}$ and $V_{C_{2}}$ from $C_{2}=V_{2} \Lambda_{C_{2}} V_{2}^{T}$. This enables to write the spectrum of $S$ as

$$
\sigma(S)=\bigcup_{r=1}^{s} \bigcup_{q=1}^{Q} \sigma\left(\hat{S}\left(\lambda_{q}, \Delta t \lambda_{r}^{\mathrm{irk}}\right)\right) \quad \lambda_{r}^{\mathrm{irk}} \in \sigma\left(A_{\mathrm{irk}}\right), \lambda_{q} \in \sigma\left(C_{2}\right),
$$

with $\hat{S}$ the matrix-valued function defined as

$$
\hat{S}(r, z)=-\left(M^{+}+z K_{1}^{+}+z r K_{2}^{+}\right)^{-1}\left(M^{-}+z K_{1}^{-}+z r K_{2}^{-}\right) .
$$

Thus, the asymptotic convergence factor of lexicographic block Gauss-Seidel applied to system (16) with $L^{*}=2$ corresponds to

$$
\rho(S)=\max _{\lambda_{\text {irk }} \in \sigma\left(A_{\text {irk }}\right)} \max _{\lambda_{q} \in \sigma\left(C_{2}\right)} \rho\left(\hat{S}\left(\lambda_{q}, \Delta t \lambda_{\text {irk }}\right)\right) .
$$

The analysis of the two-level multigrid cycle proceeds in a similar way. It is based on the matrix-valued function $\hat{T}(r, z)$ defined as

$\hat{T}(r, z)=(\hat{S}(r, z))^{s_{2}}\left(I_{N}-P_{d}\left(P_{d}^{T}\left(M+z K_{1}+z r K_{2}\right) P_{d}\right)^{-1} P_{d}^{T}\left(M+z K_{1}+z r K_{2}\right)\right)(\hat{S}(r, z))^{s_{1}}$,

with $\hat{S}(r, z)$ given by $(31)$ and $s_{1}, s_{2}$ the number of pre- and postsmoothing steps. Using this matrix function, the asymptotic convergence factor of the two-level multigrid cycle becomes

$$
\rho(T)=\max _{\lambda_{\text {irk }} \in \sigma\left(A_{\text {irk }}\right)} \max _{\lambda_{q} \in \sigma\left(C_{2}\right)} \rho\left(\hat{T}\left(\lambda_{q}, \Delta t \lambda_{\text {irk }}\right)\right) .
$$

As in the stationary case, this value corresponds to the worst case asymptotic convergence factor of multigrid applied to the set of deterministic problems

$$
\left(M+\Delta t \lambda_{\mathrm{irk}} K_{1}+\Delta t \lambda_{\mathrm{irk}} \lambda_{q} K_{2}\right) x=b \quad \text { with } \quad \lambda_{q} \in \sigma\left(C_{2}\right), \lambda_{\mathrm{irk}} \in \sigma\left(A_{\mathrm{irk}}\right) .
$$

These deterministic systems can be derived from backward Euler discretizations with scaled time step $\Delta t \lambda_{\text {irk }}$ of ODE systems

$$
M \frac{d x}{d t}+\left(K_{1}+\lambda_{q} K_{2}\right) x=b .
$$




\subsection{General discretizations with $L^{*}>2$}

The case $L^{*}=2$ enables a decoupling of the stochastic and spatial dimensions, using a similarity transform based on $C_{2}$. Hence, the analysis can be reduced to the analysis of smaller problems of the form (29) and (34). For these problems, local Fourier analysis [27, 31] allows to derive sharp convergence factors, at least for the geometric multigrid variant on regular meshes.

In general, no decoupling between the spatial and random part of the discretization is possible since the matrices $C_{i}$ cannot be diagonalized simultaneously. An exception to this occurs when double orthogonal polynomials are used as basis functions. Indeed, then all matrices $C_{i}$ are diagonal, see (2) and (7). Denoting the double orthogonal random basis by $\Psi^{\prime}$ and the corresponding matrices $C_{i}$ by $G_{i}$, we can determine the spectral radius of the two-level multigrid iteration matrix $T^{\prime}$ as

$$
\rho\left(T^{\prime}\right)=\max _{\lambda_{1}^{\prime} \in \sigma\left(G_{1}\right)} \cdots \max _{\lambda_{L^{*}}^{\prime} \in \sigma\left(G_{L^{*}}\right)} \rho\left(\hat{T}\left(\lambda_{1}^{\prime}, \ldots, \lambda_{L^{*}}^{\prime}\right)\right)
$$

with the matrix-valued function $\hat{T}\left(r_{1}, \ldots, r_{L}^{*}\right)$ defined as

$$
\begin{aligned}
\hat{T}\left(r_{1}, \ldots, r_{L^{*}}\right)= & \left(\hat{S}\left(r_{1}, \ldots, r_{L^{*}}\right)\right)^{s_{2}}\left[I_{N}-P_{d}\left(P_{d}^{T}\left(\sum_{i=1}^{L^{*}} r_{i} K_{i}\right) P_{d}\right)^{-1} P_{d}^{T}\left(\sum_{i=1}^{L^{*}} r_{i} K_{i}\right)\right] \\
& \left(\hat{S}\left(r_{1}, \ldots, r_{L^{*}}\right)\right)^{s_{1}}
\end{aligned}
$$

and the matrix-valued function $\hat{S}\left(r_{1}, \ldots, r_{L^{*}}\right)=\left(\sum_{i=1}^{L^{*}} r_{i} K_{i}^{+}\right)^{-1}\left(\sum_{i=1}^{L^{*}} r_{i} K_{i}^{-}\right)$. In Section 5 we will point out a relation between the eigenvalues of the matrices $C_{i}$ and the diagonal elements of the matrices $G_{i}$. Based on that relation, we can show that the AMG convergence properties in case of a double orthogonal random basis are similar to the case $L^{*}=2$ treated in the previous section. Moreover, also when the double orthogonal basis is not used, we can argue that the analysis of the case $L^{*}=2$ is likely to provide valuable insights for the general case $L^{*}>2$. The first terms of the system (19), i.e., $C_{1} \otimes K_{1}+C_{2} \otimes K_{2}$, represent the mean behavior of the stochastic PDE and the main stochastic variation. This follows from the stochastic discretization of the random coefficient as a truncation of a series of terms of decreasing importance, see Section 2.2. The sum involving the matrices $C_{3}, \ldots C_{L^{*}}$ can be seen as a perturbation of the system matrix. A more thorough (geometric) multigrid analysis for the general stationary case can be found in [21].

\section{A discussion of the theoretical convergence properties}

The convergence analysis of the previous section shows that both the matrices $K_{1}$ and $K_{2}$ as well as the eigenvalues of $C_{2}$ and $A_{\text {irk }}$ determine the AMG convergence, see Eq. (28) and (33). In this section we discuss the AMG convergence behavior with respect to the stochastic discretization. The conclusions agree with the properties of the geometric multigrid variant, as observed in [16] and theoretically analyzed in [21,9]. The AMG convergence behavior with respect to the IRK discretization, i.e., the influence of the eigenvalues of $A_{\text {irk }}$, is detailed in [4]. 


\subsection{A bound for the eigenvalues of $C_{2}$.}

The range of the eigenvalues of $C_{2}$ can be computed by using properties of double orthogonal random polynomials [21]. Consider the set of $Q$ generalized polynomial chaos functions $\Psi=\left[\Psi_{1}, \ldots, \Psi_{Q}\right]^{T}$. This set can be expanded to an orthonormal set $\bar{\Psi}=$ $\left[\Psi_{1}, \ldots, \Psi_{Q}, \bar{\Psi}_{Q+1}, \ldots, \bar{\Psi}_{Q^{\prime}}\right]^{T}$ that spans the same vector space as the double orthogonal polynomial chaos basis $\Psi^{\prime}$. Hence, an orthogonal matrix $Z$ exists so that $\bar{\Psi}=Z \Psi^{\prime}$. As a consequence, the matrix $\bar{C}_{2}$, defined below, can be diagonalized by the matrix $Z$,

$$
\bar{C}_{2}:=\left\langle\xi_{1} \overline{\Psi \Psi} \bar{\Psi}^{T}\right\rangle=\left\langle\xi_{1} Z \Psi^{\prime}\left(\Psi^{\prime}\right)^{T} Z^{T}\right\rangle=Z\left\langle\xi_{1} \Psi^{\prime}\left(\Psi^{\prime}\right)^{T}\right\rangle Z^{T}=Z G_{2} Z^{T} .
$$

Hence, the eigenvalues of $\bar{C}_{2}$ correspond to the diagonal entries of the diagonal matrix $G_{2}$. It can be shown that these values coincide with the roots of univariate orthogonal polynomials from the Askey scheme, as explained in [21]. Moreover, as the matrix $C_{2}$ is a principal submatrix of $\bar{C}_{2}$, the eigenvalues of $\bar{C}_{2}$ determine upper and lower bounds for the eigenvalues of $C_{2}$. This allows one to determine bounds for the eigenvalues of $C_{2}$ from the roots of certain univariate orthogonal polynomials.

\subsection{Polynomial chaos type and order.}

The influence of the polynomial chaos type and the polynomial order can be derived from the properties of the roots of the corresponding orthogonal polynomials [26]. In the case of a Legendre chaos, the eigenvalues of the matrix $C_{2}$ take values between -1 and 1 . Thus, AMG convergence is asymptotically independent of the polynomial chaos order. In case of a Hermite chaos, the eigenvalues can become arbitrarily large with a range that increases with increasing polynomial order. Whether the large eigenvalue range affects the convergence or not depends on the particular PDE problem. Consider, e.g., model problem (1) with $\alpha$ a Gaussian variable with mean 1 and variance $\nu, \alpha:=1+\sqrt{\nu} \xi_{1}$. Then, $K_{1}$ is a discretized Laplacian and $K_{2}=\sqrt{\nu} K_{1}$. According to (29), the AMG convergence corresponds to the worst convergence of multigrid applied to a set of problems of the form

$$
\left(1+\lambda_{q} \sqrt{\nu}\right) K_{1} u=b \quad \text { with } \quad \lambda_{q} \in \sigma\left(C_{2}\right), C_{2}=\left\langle\xi_{1} \Psi \Psi^{T}\right\rangle .
$$

The multiplicative factor $1+\lambda_{q} \sqrt{\nu}$ can be shifted to the right-handside. Hence, the AMG convergence is independent of the variance and of the polynomial chaos order. However, in case of $\alpha$ modelled as a random field, $\alpha(\boldsymbol{x}, \omega)$, and $L^{*}=2$, system (29) corresponds to $\tilde{K} u=b$, with $\tilde{K}$ a discretized diffusion problem with diffusion coefficient $\tilde{\alpha}(\boldsymbol{x}):=\alpha_{1}(\boldsymbol{x})+\lambda_{q} \alpha_{2}(\boldsymbol{x})$. When the ellipticity of $\tilde{\alpha}(\boldsymbol{x})$ is not satisfied, e.g. due to a large negative $\lambda_{q}$, the AMG convergence degrades severely and eventually divergence is possible. This typically occurs only for a large polynomial chaos order. For other problems, the Hermite polynomial order can have an even more serious impact on AMG convergence. For example, consider the following problem with a Gaussian random variable $\sqrt{\nu} \xi_{1}$ with zero mean and finite variance $\nu$,

$$
-\left(\frac{\partial^{2} u}{\partial x^{2}}+\left(1+\sqrt{\nu} \xi_{1}\right) \frac{\partial^{2} u}{\partial y^{2}}\right) u=b .
$$

The stiffness matrix $K_{1}$ corresponds to a discrete Laplace operator, while the second stiffness matrix $K_{2}$ only contains contributions from $\frac{\partial^{2} u}{\partial y^{2}}$. The AMG convergence rate equals the worst multigrid convergence for deterministic problems of the form

$$
-\left(\frac{\partial^{2} u}{\partial x^{2}}+\left(1+\lambda_{q} \sqrt{\nu}\right) \frac{\partial^{2} u}{\partial y^{2}}\right) u=b \quad \text { with } \quad \lambda_{q} \in \sigma\left(C_{2}\right), C_{2}=\left\langle\xi_{1} \Psi \Psi^{T}\right\rangle .
$$



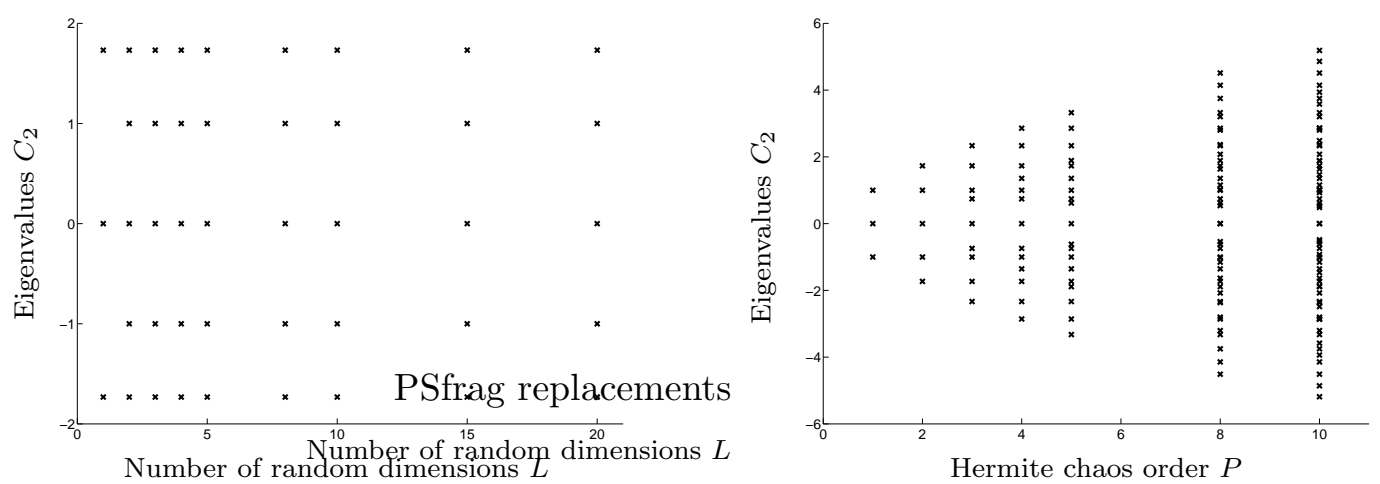

Figure 1: Effect of the polynomial chaos order and the number of random variables on the eigenvalues of $C_{2}$ (7) in case of a Hermite polynomial chaos. (a) Fixed order and increasing value of $L, P=2$. (b) Fixed number of random variables and increasing $P, L=4$.

Increasing the polynomial order broadens the range of $\lambda_{q}$ and consequently increases the anisotropy of the problem. This results in a decreased AMG convergence. Eventually, for a sufficiently large order, the problem will loose ellipticity and AMG will diverge.

\subsection{Number of random variables.}

As our convergence analysis is restricted to $L^{*}=2$, it does not provide direct information about the influence of the number of random variables $L$. However, the eigenvalue bounds of the matrices $C_{i}(7)$ do not depend on the number of random variables. This follows from the fact that the eigenvalues of $G_{i}(36)$ equal the roots of one-dimensional polynomials [21] and thus are independent of the number of random variables. This property suggests an independence of AMG convergence on the number of random variables. Fig. 1 shows the eigenvalues of $C_{2}$ as a function of the number of random variables and the polynomial chaos order.

Only in case of a double orthogonal polynomial chaos basis a quantitative analysis of $L^{*}>2$ is possible. Then the independence of AMG convergence on the number of random variables can theoretically be demonstrated.

\section{Implementation aspects}

The effectiveness of an algebraic multigrid method depends strongly on the efficiency of its implementation. In this section we point out some implementation issues that allow to reduce the computation time and memory usage.

\subsection{Matrix formulation and storage}

The tensor product formulations (19) and (16) are mathematically equivalent to the matrix systems

$$
\sum_{i=1}^{L^{*}} K_{i} U C_{i}^{T}=B \quad \text { and } \quad M X\left(C_{1} \otimes I_{s}\right)+\Delta t \sum_{i=1}^{L^{*}} K_{i} X\left(C_{i}^{T} \otimes A_{\text {irk }}^{T}\right)=\tilde{B},
$$


with the unknowns $u$ and $x$ collected in the multivectors $U \in \mathbb{R}^{N \times Q}$ and $X \in \mathbb{R}^{N \times Q s}$. This matrix representation allows an easy access of all the unknowns per nodal point: they correspond to a row in the matrix $U$ or $X$. Such access is frequently needed for the block smoothing operator, the matrix-vector multiplication in the residual computation, and the block restriction and prolongation operators. Note also that the row-by-row storage format enables a cache efficient implementation. With one memory access, a whole set of values can be retrieved from memory, that will be used in the subsequent operations.

Obviously, the entire system of dimension $N Q \times N Q$ (in the stationary case), or $N Q s \times N Q s$ (in the time-dependent case), is never stored or constructed explicitly. Only the storage of one mass matrix $M$, of $L^{*}$ stiffness matrices $K_{i}$ and $L^{*}$ matrices $C_{i}$ is required. These matrices can be stored in sparse matrix format. In general, all stiffness matrices $K_{i}$ have the same sparsity structure, hence the description of this structure has to be stored just once.

\subsection{Krylov acceleration}

Typically AMG is used as a preconditioner for a Krylov method. This makes the scheme more robust, and often significantly improves the convergence rates.

The matrix-vector multiplication needed for Krylov methods can be implemented in a cache efficient way by using the row-by-row storage format suggested above. As explained in [4], the matrix-vector product $Y=A X$ of a sparse matrix $A \in \mathbb{R}^{N \times N}$ and a multivector $X \in \mathbb{R}^{N \times Q}$ is implemented as a sequence of three nested loops where the inner loop runs over the columns of the multivectors instead of over their rows. This results in an optimal reuse of the cache since the data access patterns of $X$ and $Y$ match their storage layout.

For the stationary systems, conjugate gradients (CG) can be used as the matrices $C_{i}$ and the stiffness matrices $K_{i}$ are symmetric. In the time-dependent case, we shall use BiCGStab or one of the GMRES variants because of the non-symmetry of matrix $A_{\text {irk }}$.

\subsection{Block smoothing}

A large part of the computation time is spent in the smoothing steps. At each smoothing iteration $N$ systems of size $Q \times Q$ or $Q s \times Q s$ have to be solved. Optimizing the solution time of these local systems is therefore of utmost importance. One possible approach is to factorize these systems already during setup so that every smoothing step only matrix-vector multiplications or back substitutions are required. However, the generated fill-in may lead to excessive memory requirements for large values of $N$ and $Q$. Hence, we will not consider this further. In our experiments with direct solvers, the factorization will be done on the fly.

Depending on the properties of the local systems, different solution methods can be selected. Fig. 2 shows the average computation time of several solution approaches to solve one local system. The considered methods include an LU solver without pivoting, a sparse LU solver (UMFPACK [6] and SuperLU [7]) and a Krylov method. The tests were performed on a Pentium IV $2.4 \mathrm{GHz}$ machine with 512 MByte RAM. Values for $Q$ as a function of the number of random variables $L$ and the chaos order $P$ are given in Table 1 . This value is to be multiplied by $s$, to get the system dimension in the IRK case.

In the stationary case, considering our model problem (1) discretized with a Hermite or a Legendre chaos, the local systems (21) are sparse and symmetric positive-definite, with clustered eigenvalues and a condition number $\mathcal{O}(1)$. For large problem sizes, the CG solver leads to the best performance. No preconditioning is necessary because the systems are 


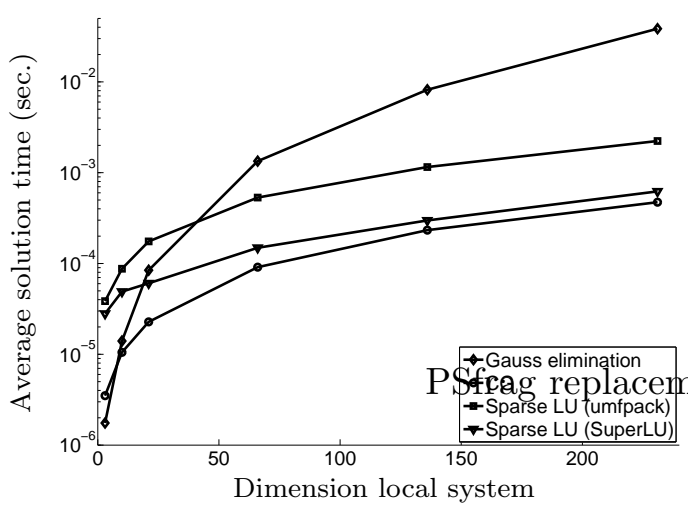

(a) Stationary problem

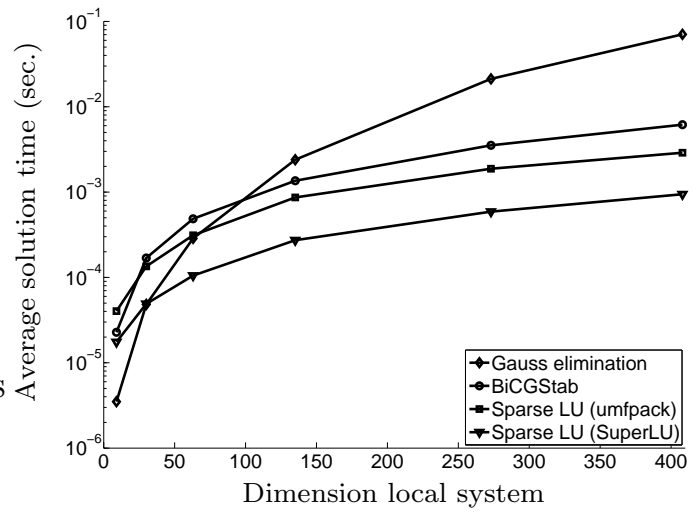

(b) Time-dependent problem

Figure 2: Average computation time to solve one local system (21) or (20) in case of the model problem (1) with $\alpha(\boldsymbol{x}, t, \omega)$ modelled as a Gaussian random field $\alpha(\boldsymbol{x}, \omega)$ with an exponential covariance function. A Hermite chaos random discretization is used and a Radau IIA IRK method.

Table 1: The number of random unknowns $Q$ as a function of the number of random variables $L$ and of the polynomial chaos order $P$.

\begin{tabular}{cccccccc}
\hline$P \backslash L$ & 1 & 2 & 4 & 8 & 10 & 15 & 20 \\
\hline 1 & 2 & 3 & 5 & 9 & 11 & 16 & 21 \\
2 & 3 & 6 & 15 & 45 & 66 & 136 & 231 \\
4 & 5 & 15 & 70 & 495 & 1001 & 3876 & 10626 \\
\hline
\end{tabular}

well conditioned. In the time-dependent case, the local systems (20) are non-symmetric and sparse. The matrices have clustered, complex eigenvalues and a condition number typically of the order $\mathcal{O}(10)$. The sparse LU solver SuperLU [7] yields the smallest execution times for non trivial problem sizes. In both the stationary and time-dependent case, a direct solver is the most efficient method if the dimension, $Q$ or $Q s$, is small enough.

When the random model parameter is discretized by a generalized polynomial chaos (4) instead of by a Karhunen-Loève expansion (5), the local systems become less sparse, see [8]. As a consequence, the local systems solves are more time consuming. The corresponding computation times for different solution methods follow however the same pattern as in Fig. 2.

\section{Numerical results}

In this section we present some numerical results obtained with the AMG method. First, we investigate the AMG convergence with respect to several discretization parameters for the stationary diffusion equation. The tests use a square spatial domain, $\mathbf{D}=[0,1]^{2}$, and piecewise linear, triangular finite elements. We consider homogeneous Dirichlet boundary conditions, and the source term $b(\boldsymbol{x}, t)$ is set to zero. The AMG prolongation operators are 
Table 2: Configurations of the random coefficient $\alpha(\boldsymbol{x}, t, \omega)$ in Eq. (1).

\begin{tabular}{llll}
\hline & Name & Random discretization & Distribution $\xi$ \\
\hline Random field & $\alpha_{\mathrm{g}}(\boldsymbol{x}, \xi)$ & Karhunen-Loève expansion & standard normal \\
& $\alpha_{\mathrm{u}}(\boldsymbol{x}, \xi)$ & Karhunen-Loève expansion & uniform on $[-1,1]$ \\
& $\alpha_{\ln }(\boldsymbol{x}, \xi)=\exp \left(\alpha_{\mathrm{g}}\right)$ & polynomial chaos expansion & standard normal \\
Random process & $\alpha_{t}(t, \xi)$ & Karhunen-Loève expansion & standard normal \\
\hline
\end{tabular}

Table 3: Number of iterations required to solve the steady-state diffusion equation corresponding to (1) with $W(2,1)$-cycles, using AMG as standalone solver, or as preconditioner for CG (between brackets).

\begin{tabular}{lccccc}
\hline $\begin{array}{l}\text { Spatial nodes } \\
Q=21, P=2, L=5\end{array}$ & $N=10177$ & $N=50499$ & $N=113981$ & $N=257488$ & $N=356806$ \\
\hline$\alpha_{\mathrm{g}}$ & $31(15)$ & $35(16)$ & $36(17)$ & $36(17)$ & $37(17)$ \\
$\alpha_{\mathrm{u}}$ & $31(15)$ & $34(16)$ & $36(17)$ & $36(17)$ & $37(17)$ \\
$\alpha_{\ln }$ & $32(16)$ & $37(17)$ & $39(18)$ & $38(17)$ & $39(18)$ \\
\hline \hline Random variables & $L=1$ & $L=5$ & $L=10$ & $L=15$ & $L=20$ \\
$N=20611, P=2$ & $Q=3$ & $Q=21$ & $Q=66$ & $Q=136$ & $Q=231$ \\
\hline$\alpha_{\mathrm{g}}$ & $32(15)$ & $34(16)$ & $34(16)$ & $35(16)$ & $35(16)$ \\
$\alpha_{\mathrm{u}}$ & $32(15)$ & $33(16)$ & $34(16)$ & $35(16)$ & $35(16)$ \\
$\alpha_{\ln }$ & $35(16)$ & $36(17)$ & $36(17)$ & $36(17)$ & $37(17)$ \\
\hline \hline Chaos order & $P=1$ & $P=2$ & $P=3$ & $P=4$ & $P=5$ \\
$N=20611, L=5$ & $Q=6$ & $Q=21$ & $Q=56$ & $Q=126$ & $Q=252$ \\
\hline$\alpha_{\mathrm{g}}$ & $33(15)$ & $34(16)$ & $34(16)$ & $35(16)$ & $36(17)$ \\
$\alpha_{\mathrm{u}}$ & $33(15)$ & $33(16)$ & $34(16)$ & $35(16)$ & $35(17)$ \\
$\alpha_{\ln }$ & $34(16)$ & $36(17)$ & $37(17)$ & $37(17)$ & $38(18)$ \\
\hline
\end{tabular}

built with classical Ruge-Stüben AMG [24]. The stopping criteria for the AMG method is a residual norm smaller than $10^{-10}$. A random initial approximation to the solution was used. We consider several configurations for the random input $\alpha(\boldsymbol{x}, t, \omega)$. For each case, Table 2 indicates which expansion is used to construct the random input and what type of random variables are present in that expansion. In case of a Karhunen-Loève expansion, an exponential covariance function is assumed, $C_{\alpha \alpha}\left(\boldsymbol{x}, \boldsymbol{x}^{\prime}\right)=\nu \exp \left(-\frac{\left|\boldsymbol{x}-\boldsymbol{x}^{\prime}\right|}{l_{c}}\right)$, with variance $\nu=0.1$ and correlation length $l_{c}=1$. In case of the lognormal random field $\alpha_{\ln }$, the variance of the underlying Gaussian field $\alpha_{g}$ equals 0.3. When the stochastic discretization is based on uniformly distributed random variables, a Legendre polynomial chaos is used, in case of standard normal distributed random variables a Hermite chaos. For each configuration of $\alpha$, the mean value of the random input always equals the constant function 1 . Next, the AMG performance will be illustrated for a more complex test problem.

\subsection{Stationary problems}

The dependence of the AMG convergence properties on the spatial and stochastic discretization parameters is illustrated by the numerical results displayed in Table 3. As expected from 

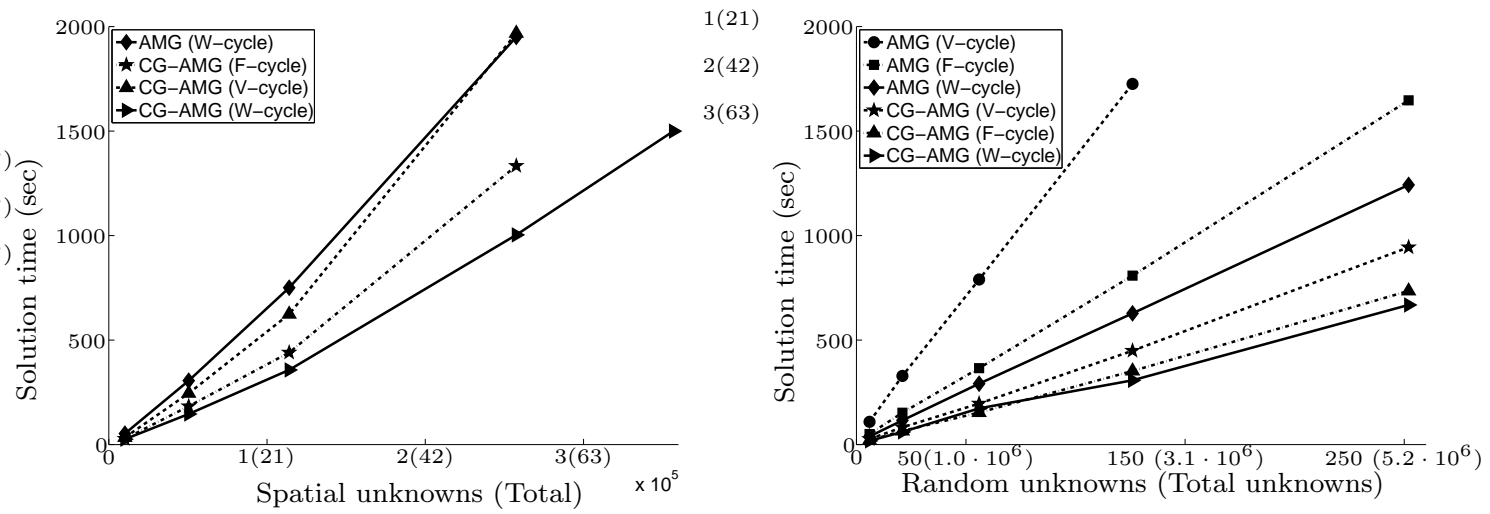

Figure 3: Total solution time when solving the steady state problem with $\alpha_{\mathrm{u}}(\boldsymbol{x}, \xi), L=5$ and $(2,1)$-cycles of AMG iterations. (a) A second-order Legendre chaos is used, resulting in $Q=21$. (b) The discretization is based on a first until a fifth order Legendre chaos, and a mesh with 20611 nodes.

the discussion in Section 5, the number of AMG iterations is independent of the stochastic and spatial discretization when applied to our model problem. The independence on the polynomial case order is maintained in case of a Hermite chaos for a low to moderate polynomial chaos order. Applying Krylov acceleration results in more robust convergence and reduced computing times.

The computation times for the calculations in Table 3 are presented graphically in Fig. 3. The total AMG solution time is shown as a function of the number of spatial nodes and of the number of random unknowns. For this problem the matrices $C_{i}$ are defined as in Eq. (7). By increasing the number of spatial nodes, the number of local solves in the blocksmoother increases proportionally. In addition, extra coarse levels may be introduced so that the total increase in computation time is no longer linear. The results from the right figure were obtained by increasing the polynomial chaos order while keeping the number of random variables $L$ constant. Thus, only the dimension of the matrices $C_{i}$ increases; the number of stiffness matrices $L^{*}$ remains the same. This mainly affects the cost of the block solves in the smoother. With CG, the number of iterations required is proportional to the square root of the condition number of (21). In practice this condition number is close to 1 so that the number of CG iterations is more or less independent of the dimension of the systems. The cost of each CG iteration depends on the sparsity of the matrix, which, with $C_{i}$ defined by (7), is of the order $\mathcal{O}(Q)$. This result in a cost $\mathcal{O}(Q)$ to solve one block system in the smoother. The linear increase of the computation time in function of the number of random unknowns $Q$ is clearly observed in Fig. 3. If the number of stiffness matrices is also increased, then the total computing time tends to grow faster than linear. Also in case of a polynomial chaos expansion of the random input, as in the lognormal field example, higher computing times are observed. This is caused by the larger number of stiffness matrices, $Q$ instead of $L+1$, and by the decreased sparsity of the matrices $C_{i}(8)$.

As discussed in Section 5, the convergence analysis indicates that the convergence of AMG is asymptotically independent of the polynomial chaos order in case of a Legendre chaos, but not in case of a Hermite chaos. For model problem (1), only a large polynomial chaos order 
Table 4: Number of iterations required to solve problem $(38)$ with W(2,1)-cycles, using AMG as standalone solver, or as preconditioner for CG (between brackets), until $\|$ residual $\|<10^{-10}$. The finite element mesh consists of $N=20611$ nodes, and 5 random variables are used to discretize the random space.

\begin{tabular}{lccccc}
\hline Polynomial chaos order & $P=1$ & $P=2$ & $P=3$ & $P=4$ & $P=5$ \\
\hline$\alpha_{\mathrm{g}}$ & $24(16)$ & $25(17)$ & $27(19)$ & $35(22)$ & $86(61)$ \\
$\alpha_{\mathrm{u}}$ & $24(16)$ & $24(16)$ & $25(17)$ & $25(17)$ & $25(17)$ \\
$\alpha_{\ln }$ & $43(25)$ & $48(28)$ & $58(33)$ & $72(40)$ & $97(53)$ \\
\hline
\end{tabular}

Table 5: Number of iterations required to solve the time-dependent problem (1) with $V(2,1)$-cycles, using AMG as standalone solver, or as preconditioner for BiCGStab (between brackets). The discretization is based on a finite element mesh with 20611 nodes, a secondorder Hermite chaos with $L=5$, corresponding to $Q=21$, and a Radau II A implicit Runge-Kutta scheme with $\Delta t=0.01$.

\begin{tabular}{lcccccc}
\hline Time discretization order & 1 & 3 & 5 & 7 & 9 & 11 \\
IRK stages & $s=1$ & $s=2$ & $s=3$ & $s=4$ & $s=5$ & $s=6$ \\
\hline$\alpha_{\mathrm{g}}$ & $41(18)$ & $33(19)$ & $29(19)$ & $27(20)$ & $27(18)$ & $27(19)$ \\
$\alpha_{\mathrm{t}}$ & $39(18)$ & $32(19)$ & $28(19)$ & $27(18)$ & $27(19)$ & $27(19)$ \\
\hline
\end{tabular}

has an impact on the multigrid convergence. For some problems however, also small values of the polynomial chaos order affect the AMG convergence rate. This is illustrated by the problem

$$
\frac{\partial^{2} u(\boldsymbol{x}, \omega)}{\partial x^{2}}+\alpha(\boldsymbol{x}, \omega) \frac{\partial^{2} u(\boldsymbol{x}, \omega)}{\partial y^{2}}=0,
$$

which is discretized similarly as our model problem. Table 4 illustrates the deteriorating AMG convergence when increasing the polynomial chaos order in case of a Hermite chaos. As expected, the number of iterations remains unchanged in case of a Legendre chaos.

\subsection{Time-dependent problems}

First, we illustrate the influence of the number of IRK-stages on the AMG convergence, for model problem (1). The results are presented in Table 5 for a number of stages $s$, increasing from 1 up to 6 . The first corresponds to a first-order method, while the later leads to a time-integration scheme of order 11. As a test case, a Gaussian field $\alpha_{g}(\boldsymbol{x}, \xi)$ and a Gaussian process $\alpha_{t}(t, \xi)$ were used, see Table 2 for the characteristics. Note that the convergence analysis predicts an increased AMG convergence rate when the number of IRK stages is increased. This effect is visible in the numerical results.

Finally, we consider a more challenging transient potential equation,

$$
\frac{\partial V(\mathbf{x}, t, \omega)}{\partial t}-\nabla \cdot(\varepsilon(\omega) \nabla V(\mathbf{x}, t, \omega))=0,
$$

on a complex domain. Here, $V$ denotes the electric potential and $\varepsilon$ the electric permittivity. No charge density $\rho$ is present. The domain, the boundary conditions and the setup for $\varepsilon$ are 


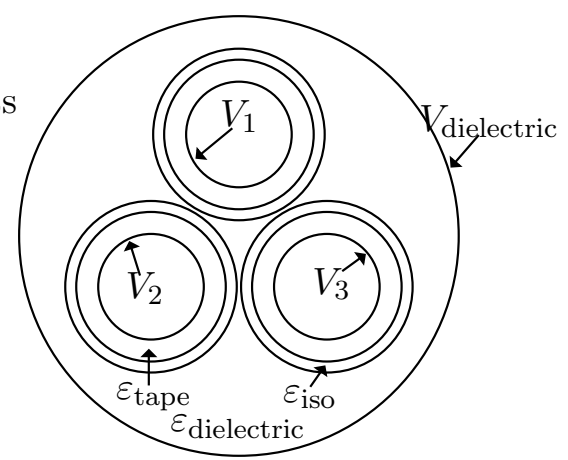

\begin{tabular}{lr}
\hline boundary conditions & \\
\hline$V_{\text {dielectric }}$ & 0 \\
$V_{1}$ & 0.087 \\
$V_{2}$ & 0.819 \\
$V_{3}$ & -0.906 \\
\hline electric permittivity & \\
\hline$\varepsilon_{\text {dielectric }}$ & $\varepsilon_{0}\left(1+0.3 \xi_{1}\right)$ \\
$\varepsilon_{\text {iso }}$ & $24 \varepsilon_{0}\left(1+0.1 \xi_{2}\right)$ \\
$\varepsilon_{\text {tape }}$ & $6 \varepsilon_{0}\left(1+0.2 \xi_{3}\right)$ \\
\hline
\end{tabular}

Figure 4: Configuration of a $2 \mathrm{D}$ stochastic problem. The random variables $\xi_{1}, \xi_{2}, \xi_{3}$ are independent and each uniformly distributed on $[-1,1]$. The permittivity of the free space, $\varepsilon_{0}=8.85 \cdot 10^{-12}$.

presented in Fig. 4. The model represents a three-phase cable, with four constant potentials along the outer and inner boundaries. Fig. 5 shows the mean value and the variance of the electric potential at several instances in time. Applying AMG results in similar convergence properties as the ones in our earlier experiments. An illustration of the convergence history as a function of the iteration index is given in Fig. 6. Observe that the use of GMRESR [28] results in a more robust convergence than BiCGStab. This is typically also the case for classical deterministic PDEs. To limit the memory requirements of GMRESR, the method is restarted every 5 iterations.

\section{Conclusions}

We have constructed and analyzed an algebraic multigrid method for stochastic finite element discretizations of time-dependent stochastic PDEs. This work extends previous research on multigrid for stochastic finite element problems [21,9] towards unstructured finite element meshes and high-order time discretizations. The presented AMG method has very favorable convergence properties with respect to the spatial, random and time discretization.

In order to solve real engineering stochastic PDEs by the stochastic finite element method however, further research is necessary. By using the knowledge of the stochastic discretization, the AMG components may be enhanced and optimized. Also, when solving large-scale realistic problems a parallel implementation will become essential.

\section{Appendix}

\section{The computation of $\left\langle\Psi_{i} \Psi_{j} \Psi_{k}\right\rangle$ in case of a Hermite chaos}

The Hermite chaos of order $P$ and defined over $L$ standard normal variables $\xi_{1}, \ldots, \xi_{L}$ is constructed as a set of $Q$ multivariate Hermite polynomials $\Psi_{q}$, each defined as [18]

$$
\Psi_{q}=\prod_{i=1}^{L} \frac{1}{\sqrt{\eta_{q, i} !}} H_{\eta_{q, i}}\left(\xi_{i}\right)
$$



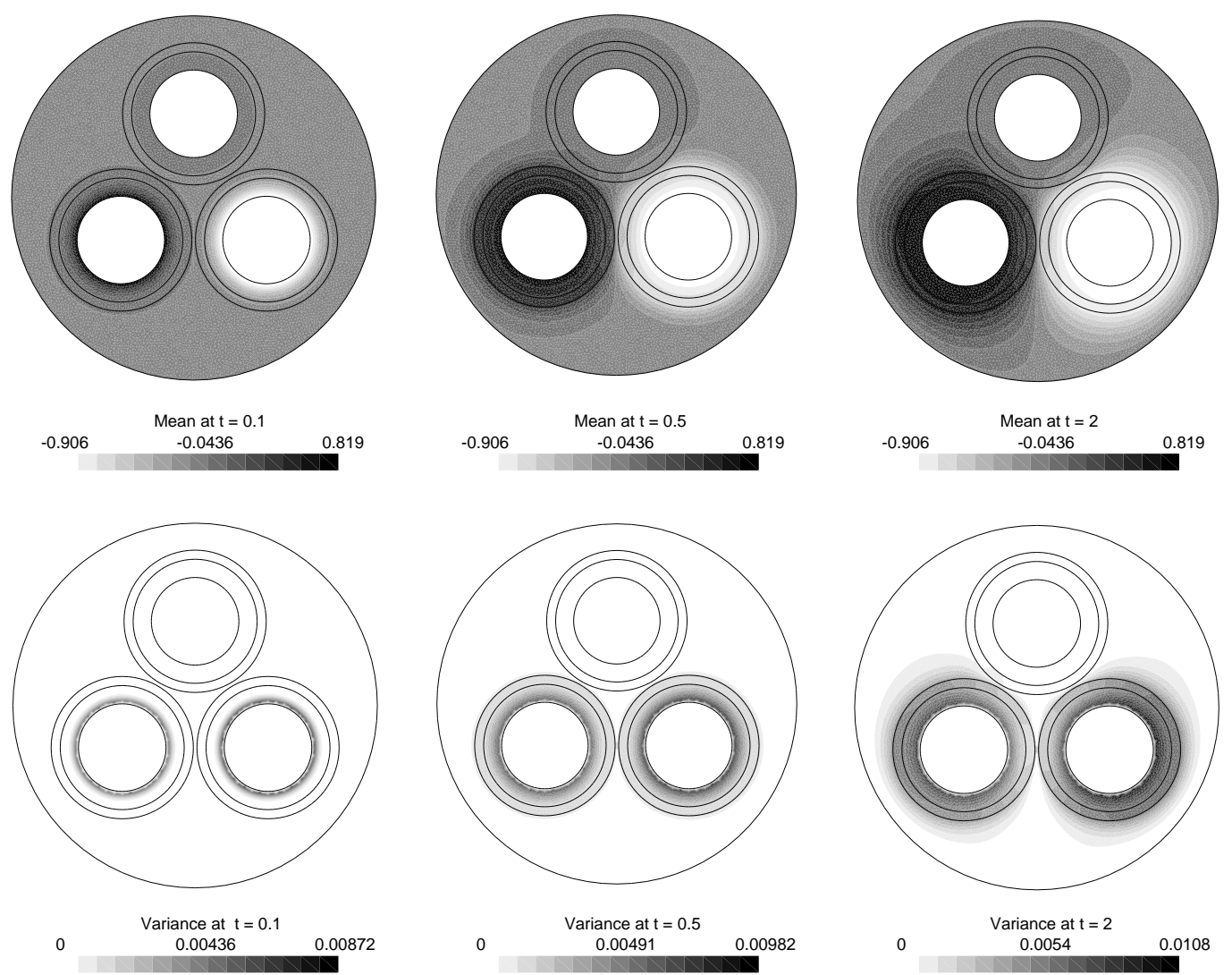

Figure 5: Mean and variance of the solution of Eq. (39). The configuration of Fig. 4 is used with a two-stage Radau IIA IRK discretization and time step 0.05. The electric potential is zero initially.

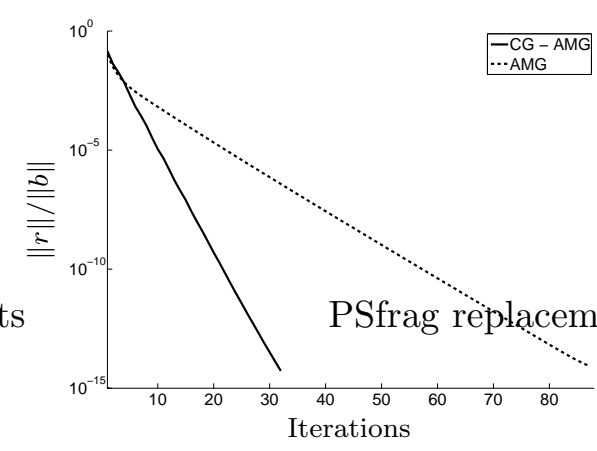

(a) Steady-state problem, F(2,1) cycles

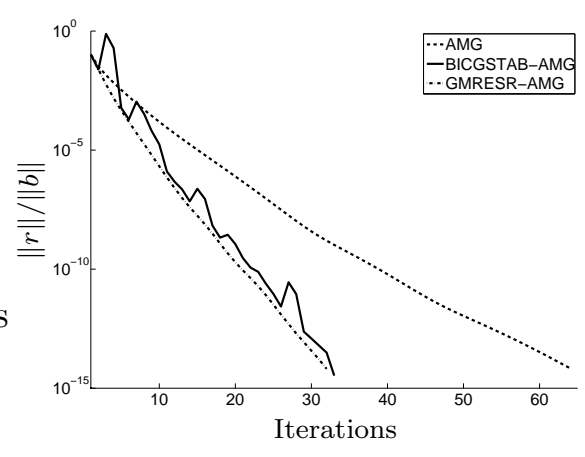

(b) Transient problem, V(2,1) cycles

Figure 6: Residual norms as a function of the number of iterations when solving Eq. (39) on the domain represented by Fig. 4, discretized with 166245 nodes. A 3-stage Radau IIA IRK discretization with time step 0.05 is used together with a second-order Legendre chaos resulting in a total of $1.7 \cdot 10^{6}$ unknowns in the steady-state case and $5.0 \cdot 10^{6}$ in the timedependent case. 
with $H_{n}(z)$ a one-dimensional Hermite polynomial of order $n$ and $\eta_{q}=\left(\eta_{q, i}, \ldots, \eta_{q, L}\right)$ a set of non-negative integers with only a finite number non-zeros and $\sum_{i=1}^{L} \eta_{q, i}<P$. The factor $1 / \sqrt{\eta_{q, i} !}$ guarantees the normalization of the multivariate polynomials. The onedimensional polynomials $H_{n}(z)$ are recursively defined as [23]

$$
H_{0}(z)=1, \quad H_{1}(z)=z, \quad H_{n+1}(z)=z H_{n}(z)-n H_{n-1}(z) .
$$

Based on the properties of Hermite polynomials [26, p. 390], the inner product of three multivariate Hermite polynomials can be calculated as

$$
\left\langle\Psi_{i} \Psi_{j} \Psi_{k}\right\rangle=\prod_{m=1}^{L} \frac{\sqrt{\eta_{i m} !} \sqrt{\eta_{j m} !} \sqrt{\eta_{k m} !}}{\left(s_{m}-\eta_{i m}\right) !\left(s_{m}-\eta_{j m}\right) !\left(s_{m}-\eta_{k m}\right) !},
$$

if $2 s_{m}=\eta_{i m}+\eta_{j m}+\eta_{k m}$ is an even integer and $s_{m} \geq \eta_{i m}, s_{m} \geq \eta_{j m}, s_{m} \geq \eta_{k m}$, otherwise the inner product is zero.

\section{References}

[1] I. Babuška and P. Chatzipantelidis. On solving elliptic stochastic partial differential equations. Comput. Methods Appl. Mech. Engrg., 191:4093-4122, 2002.

[2] I. Babuška, R. Tempone, and G. Zouraris. Galerkin finite element approximations of stochastic elliptic partial differential equations. SIAM J. Numer. Anal., 42:800-825, 2004 .

[3] I. Babuška, R. Tempone, and G. E. Zouraris. Solving elliptic boundary value problems with uncertain coefficients by the finite element method: the stochastic formulation. Comput. Methods Appl. Mech. Engrg., 194:1251-1294, 2005.

[4] T. Boonen, J. Van lent, and S. Vandewalle. An algebraic multigrid method for high order time-discretization of the div-grad and the curl-curl equations. Technical Report TW483, Katholieke Universiteit Leuven, 2006.

[5] A. Brandt. Multi-level adaptive solutions to boundary-value problems. Math. Comput., 31:333-390, 1977.

[6] T. A. Davis. Algorithm 832: UMFPACK V4.3 - an unsymmetric-pattern multifrontal method. ACM Trans. Math. Softw., 30(2):196-199, 2004.

[7] J. W. Demmel, S. C. Eisenstat, J. R. Gilbert, X. S. Li, and J. W. H. Liu. A supernodal approach to sparse partial pivoting. SIAM J. Matrix Analysis and Applications, 20(3):720-755, 1999.

[8] M. Eiermann, O. G. Ernst, and E. Ullmann. Computational aspects of the stochastic finite element method. Computing and visualization in science, 10(1):3-15, 2007.

[9] H. Elman and D. Furnival. Solving the stochastic steady-state diffusion problem using multigrid. Technical report TR-4786, University of Maryland, Departement of Computer Science, March 2007. 
[10] R. Ghanem. The nonlinear Gaussian spectrum of log-normal stochastic processes and variables. J. Appl. Mech. Trans. ASME, 66(4):964-973, 1999.

[11] R. Ghanem, G. Saad, and A. Doostan. Efficient solution of stochastic systems: application to the embankment dam problem. Structural safety, 29(3):238-251, 2007.

[12] R. Ghanem and P. Spanos. A spectral stochastic finite element formulation for reliability analysis. J. Engrg. Mech. ASCE, 17:2351-2372, 1991.

[13] R. Ghanem and P. Spanos. Stochastic finite elements, a spectral approach. Dover Publications, Mineola, New York, 2003.

[14] E. Hairer and G. Wanner. Solving ordinary differential equations II: stiff and differentialalgebraic problems. Springer-Verlag, Berlin, Germany, 1991.

[15] G. Karniadakis, C.-H. Su, D. Xiu, D. Lucor, C. Schwab, and R. Todor. Generalized polynomial chaos solution for differential equations with random inputs. Research Report 2005-01, Seminar for Applied Mathematics, ETH Zürich, January 2005.

[16] O. Le Maître, O. Knio, B. Debusschere, H. Najm, and R. Ghanem. A multigrid solver for two-dimensional stochastic diffusion equations. Comput. Methods Appl. Mech. Engrg., 192:4723-4744, 2003.

[17] M. Loève. Probability theory. Springer, New York, USA, 1977.

[18] H. Matthies and A. Keese. Galerkin methods for linear and nonlinear elliptic stochastic partial differential equations. Comput. Methods Appl. Engrg., 194:1295-1331, 2005.

[19] K. Phoon, S. Huang, and S. Quek. Simulation of second-order processes using KarhunenLoève expansion. Computers and Structures, 80:1049-1060, 2002.

[20] G. I. Schuëller. A state-of-the-art report on computational stochastic mechanics. Prob. Engrg. Mech., 12(4):197-322, 1997.

[21] B. Seynaeve, E. Rosseel, B. Nicolaï, and S. Vandewalle. Fourier mode analysis of multigrid methods for partial differential equations with random coefficients. Journal of Computational Physics, 224:132-149, 2007.

[22] M. Shinozuka and G. Deodatis. Response variability of stochastic finite element systems. J. Eng. Mech., 114:499-519, 1988.

[23] C. Soize and R. Ghanem. Physical systems with random uncertainties: chaos representations with arbritary probability measure. SIAM J. Sci. Comput., 26(2):395410, 2004.

[24] K. Stüben. A review of algebraic multigrid. Journal of Computational and Applied Mathematics, 128:281-309, 2001.

[25] B. Sudret and A. Der Kiureghian. Stochastic finite elements and reliability: a stateof-the-art report. Technical Report UCB/SEMM-2000/08, University of California, Berkeley, 2000. 
[26] G. Szegö. Orthogonal polynomials. American Mathematical Society Colloq. Publ., Providence, USA, $4^{\text {th }}$ edition, 1967.

[27] U. Trottenberg, C. Oosterlee, and A. Schüller. Multigrid. Academic Press, San Diego, USA, 2001.

[28] H. Van der Vorst and C. Vuik. GMRESR: a family of nested GMRES methods. Numerical Linear Algebra with Applications, 1(4):369-386, 1994.

[29] J. Van lent and S. Vandewalle. Multigrid methods for implicit Runge-Kutta and boundary value method discretizations of parabolic pdes. SIAM J. Sci. Comput., 27(1):67-92, 2005.

[30] X. Wan and G. E. Karniadakis. An adaptive multi-element generalized polynomial chaos method for stochastic differential equations. J. Comput. Phys., 209(2):617-642, 2005.

[31] R. Wienands and W. Joppich. Practical Fourier analysis for multigrid methods. CRC Press, Boca Raton, Florida, USA, 2005.

[32] D. Xiu and G. Karniadakis. Modeling uncertainty in steady state diffusion problems via generalized polynomial chaos. Comput. Methods Appl. Mech. Engrg., 191:4927-4948, 2002.

[33] D. Xiu and G. Karniadakis. The Wiener-Askey polynomial chaos for stochastic differential equations. SIAM J. Sci. Comput., 24(2):619-644, 2002.

[34] D. Xiu and G. Karniadakis. Modeling uncertainty in flow simulations via generalized polynomial chaos. Journal of Computational Physics, 187:137-167, 2003. 